\title{
Calculating statistical power for meta-analysis using metapower
}

\author{
Jason W. Griffin $\mathrm{a} \bowtie \bullet$ \\ ${ }^{\mathrm{a}}$ Department of Psychology, Pennsylvania State University
}

\begin{abstract}
Meta-analysis is an influential evidence synthesis technique that summarizes a body of research. Though impactful, meta-analyses fundamentally depend on the literature being sufficiently large to generate meaningful conclusions. Power analysis plays an important role in determining the number of studies required to conduct a substantive meta-analysis. Despite this, power analysis is rarely conducted or reported in published meta-analyses. A significant barrier to the widespread implementation of power analysis is the lack of available and accessible software for calculating statistical power for meta-analysis. In this paper, I provide an introduction to power analysis and present a practical tutorial for calculating statistical power using the R package metapower. The main functionality includes computing statistical power for summary effect sizes, tests of homogeneity, categorical moderator analysis, and subgroup analysis. This software is free, easy-to-use, and can be integrated into a continuous work flow with other meta-analysis packages in R.
\end{abstract}

Keywords m moderator analysis, power analysis, statistical software, systematic review, evidence synthesis. Tools $\square \mathrm{R}$.

\section{jxg569@psu.edu}

10.20982/tqmp.17.1.p024

\author{
Acting Editor \\ Roland Pfister (Uni- \\ versität Würzburg) \\ Reviewers \\ - One anonymous re- \\ viewer.
}

\section{Introduction}

Meta-analysis is a powerful statistical tool widely used across a broad range of scientific disciplines to quantitatively summarize an area of research. By identifying, synthesizing, and summarizing empirical research findings, meta-analyses increase generalizability and improve effect size estimates of the existing literature. Evidence synthesis methods like meta-analysis are widely regarded as the highest form of scientific evidence, and routinely inform policy decisions, clinical practice, and evidencebased medicine (Gopalakrishnan \& Ganeshkumar, 2013). Although meta-analyses are highly influential, this technique is resource intensive and time-consuming, often taking at least a year to complete (Borah, Brown, Capers, \& Kaiser, 2017). In addition to logistical demands, conducting a meta-analysis before enough studies are available can result in inaccurate and misleading conclusions, especially when the number of studies is small (Jackson \& Turner, 2017; Thorlund et al., 2011). Therefore, power analysis plays an important role in the planning stage and is nec- essary to determine the feasibility of a meta-analysis.

The goal of power analysis in primary and metaanalytic research is to determine the number of participants or number of studies, respectively, needed to have a reasonable chance at rejecting the null hypothesis given a statistical test. For meta-analysis, these statistical tests include estimating a summary effect size that is different than zero (e.g., size of association between variables, group differences on a variable), evaluating whether there are group differences in effect size between different types of studies (e.g., children vs. adult studies), or evaluating whether there are subgroup differences in effect size within studies (e.g., men vs. women). By postulating what we expect to find, it becomes possible to calculate statistical power - the probability of rejecting the null hypothesis when, in fact, the alternative hypothesis is true.

For primary research, power analysis is used at the planning and design stage of a study to determine the number of participants required to detect a substantive effect given an expected effect size. Widely considered an essential part of research design, apriori power analyses are 
often required for federally funded research grants and randomized controlled trials (National Institute of Health, 2018). The inclusion of power analysis in evidence synthesis methods like meta-analysis is also critical because underpowered meta-analyses lack precision in estimating a summary effect size - especially when individual studies vary considerably - which can lead to conclusions that are incorrect (Thorlund et al., 2011; Pigott \& Polanin, 2020; Jackson \& Turner, 2017). Although the necessary underlying theoretical statistics, equations, and procedures have been articulated (Hedges \& Pigott, 2001, 2004; Jackson \& Turner, 2017; Pigott, 2012; Valentine, Pigott, \& Rothstein, 2009), power analyses are rarely considered, conducted, or reported in published meta-analyses.

A significant barrier to the widespread implementation of power analysis in meta-analysis is the lack of easyto-use software. While numerous software options like $\mathrm{G}^{*}$ power have been developed to compute power calculations for primary research, allowing for widespread implementation of power analysis in primary research (Faul, Erdfelder, Lang, \& Buchner, 2007), analogous software options do not exist for meta-analysis, despite similarity in procedure. This means that to compute statistical power for meta-analysis, researchers must manually perform the calculations, use an online calculator, or utilize a userdefined script (e. g., Cafri, Kromrey, \& Brannick, 2009). Such resources can be limited in functionality and difficult to integrate into a reproducible work flow.

To fill this methodological gap, I developed metapower, an $\mathrm{R}$ package for computing statistical power for metaanalysis. This package supports power analysis for (1) summary effect sizes; (2) tests of homogeneity; (3) moderator analysis; and (4) subgroup analysis. Additionally, power calculations are available for fixed- and randomeffects models and can accommodate multiple types of effect sizes (i. e., Cohen's $d$, correlation coefficient, and odds ratio). metapower was designed to be user-friendly (i. e., minimal coding) and accessible (i. e., free) to researchers with various degrees of expertise including students, principal investigators, applied researchers, non-statisticians, and those with little programming experience. To compliment this goal, I also developed a fully functional, web-based application for users unfamiliar with R (jasongriffin.shinyapps.io/shiny_metapower). In what follows, I overview the major components of power analysis for meta-analysis, provide guidance on how to make decisions about anticipated parameter values, and provide a step-bystep tutorial on how to conduct a power analysis for metaanalysis using metapower.

\section{Power analysis for meta-analysis}

Like traditional power analysis, computing statistical power for a meta-analysis requires making informed assumptions about expected findings. In primary research, this includes the effect size magnitude and sample size of an individual study. For meta-analysis, the unit of analysis is an individual study (rather than a participant); therefore, power is calculated based on expected values for effect size magnitude, sample size, the number of studies, and the amount of between-study variability. Generally speaking, power can be calculated with four values: effect size magnitude, study sample size, number of studies, degree of heterogeneity.

\section{Study-specific effect sizes and variances}

Meta-analyses can be conducted using different types of effect sizes, including Cohen's $d$, correlation coefficient, and odds ratio (Pigott, 2012). Importantly, different effect size metrics generally reflect specific types of research questions. For example, the mean difference between two independent groups can be evaluated with Cohen's $d$, whereas a correlation coefficient reflects the within-group association between two continuous variables. The logic for calculating statistical power is similar for each of these as the input data for meta-analysis are the study-specific effect sizes and variances. However, the distributional characteristics for some effect sizes are unfavorable for quantitative synthesis and must be transformed for meta-analysis and as a consequence, for power analysis as well. Cohen's $d$ can be used directly in meta-analysis as computed with

$$
\begin{gathered}
E S_{d}=\frac{M_{2}-M_{1}}{s_{p}} \\
v_{d}=\frac{n_{1}+n_{2}}{n_{1} n_{2}}+\frac{E S_{d}^{2}}{2\left(n_{1}+n_{2}\right)}
\end{gathered}
$$

where $M_{2}$ and $M_{1}$ are the two group means and $s_{p}$ is the pooled standard deviation. In contrast, the correlation coefficient, $r$, is not normally distributed; thus, meta-analysis of correlation coefficients use Fisher's r-to-z transformations and the respective variance given by

$$
\begin{gathered}
E S_{r z}=0.5 \ln \left[\frac{1+r}{1-r}\right] \\
v_{r z}=\frac{1}{n-3}
\end{gathered}
$$

Odds ratios are also not normally distributed and range from 0 to $\infty$. As a result, meta-analyses of odds ratios are conducted using the log odds ratio, $E S_{l o g(O R)}$, and respective variance, $v_{\log (O R)}$, given by

$$
E S_{\log (O R)}=\ln \left(\frac{a d}{b c}\right)
$$




$$
v_{\log (O R)}=\frac{1}{a}+\frac{1}{b}+\frac{1}{c}+\frac{1}{d}
$$

Throughout the remainder of this paper, equations will refer to effect size, $E S$, and variance, $v$, whose values depend on the type of effect size as shown above.

\section{Power for summary effect size}

In meta-analysis, input data reflects study-specific effect sizes $\left(E S_{i}\right)$ and variances $\left(v_{i}\right)$ for $i$ th study where $i=1, \ldots$, $k$, where $k$ is the total number of studies. These input data are statistically combined to estimate a weighted summary effect size $\theta$ and variance $v_{\text {. }}$. The goal of power analysis is to determine the probability of correctly rejecting the null hypothesis (e.g., $\theta=0$ ) in favor of an expected alternative, where the alternative is based on what researchers expect to find (e.g., $\theta=0.5$ ). Since providing guesses about each study-specific effect size and variance is nonviable, it is assumed that overall effect size, $E S=E S_{i}$, and overall variance, $v=v_{i}$, for $i$ th study where $i=1, \ldots, k$. Furthermore, since $v$ depends on $v_{i}$ and within-study sample sizes $n_{i}$, power calculations also assume that studies have the same sample sizes, such that $n=n_{i}$ for $i$ th study where $i=1, \ldots, k$. In other words, prior expectations should be based on a "typical" study that is most characteristic of the prospective meta-analysis (Hedges \& Pigott, 2001; Jackson \& Turner, 2017).

\section{Fixed-effects model}

Fixed-effects models assume that a single common effect size underlies all study-specific effect sizes in a metaanalysis. To calculate power under this assumption, we first posit a value for the expected overall effect size, $E S$, within-study sample size, $n$, and the total number of studies to be included in the meta-analysis, $k$ (Hedges \& Pigott, 2001). With these expected values, it is possible to derive an alternative distribution representing the expected outcome, which can be compared to the null distribution. This alternative is given by the non-centrality parameter, $\lambda$, which is based on the value of the expected summary effect size $\theta$ and variance $v_{\bullet}$. The weighted summary effect size reflects the expected magnitude of the effect size such that $\theta=E S$, whereas the weighted variance $v_{\bullet}=v / k$, where $v$ reflects the the common variance (see Eq. 2, 4, \& 6 ), and $k$ is the total number of studies (Hedges \& Pigott, 2001). With $\theta$ and $v_{\bullet}$, the non-centrality parameter $\lambda$ can be calculated with

$$
\lambda=\frac{\theta-0}{\sqrt{v_{\bullet}}}
$$

This non-centrality parameter can then be compared to the null distribution to derive the probability of rejecting the null hypothesis in favor of the expected alternative. For a summary effect size, the null hypothesis is that the summary effect size equals zero $\left(H_{0}: \theta=0\right)$, and is based on a standard normal distribution $(M=0, S D=1)$, whereas the expected alternative distribution has a mean equal to $\lambda$ and variance 1 . The statistical power for this test is given by calculating the area under the alternative distribution that exceeds the critical value of the null distribution written as

$$
\text { power }=1-\Phi\left(c_{\alpha}-\lambda\right)
$$

where $\Phi(x)$ is the cumulative distribution function, $c_{\alpha}$ is the specified critical value, and $\lambda$ is the non-centrality parameter. For a two-tailed test, the area under the curve that is greater or less than $\left|c_{\alpha / 2}\right|$ is written as

$$
\text { power }=1-\Phi\left(c_{\alpha / 2}-\lambda\right)+\Phi\left(c_{\alpha / 2}-\lambda\right)
$$

\section{Random-effects model}

Power analysis for fixed-effects models relies on the strict assumption that a single effect size underlies all individual studies included in the meta-analysis. This strong assumption is difficult to justify in the majority of cases because studies vary considerably with regard to their population, inclusion criteria, methodology, and measurement. Under random-effects models, power analysis is more complex because variation among effect sizes is the result of withinstudy variance, $v$, and the estimated between-study variability, $\tau^{2}$. To account for this additional source of variation, the conventional approach has been to posit different values of $\tau^{2}$ and incorporate into the weighted variance $v_{\bullet}=\left(v+\tau^{2}\right) / k$. However, $\tau^{2}$ is a parameter that is estimated with some degree of uncertainty and the aforementioned method does not account for this uncertainty. Importantly, when $\tau^{2}$ is estimated with large uncertainty (e.g., in the case of meta-analysis with few studies), statistical power is reduced compared to when $\tau^{2}$ is estimated with greater certainty. Considering the median number of studies included in meta-analyses is estimated to be three (Davey, Turner, Clarke, \& Higgins, 2011), accounting for the uncertainty of $\tau^{2}$ is important for obtaining accurate power calculations (Jackson \& Turner, 2017).

To account for uncertainty in the estimation of $\tau^{2}$, Jackson and Turner (2017) developed an approach to compute statistical power for random-effects meta-analysis that only requires researchers to posit values for the summary effect size, within-study sample size, total number of studies, and degree of between-study variability (i. e., $\tau^{2}$ ). However, numerical estimates of $\tau^{2}$ are difficult to interpret by themselves especially apriori; therefore, it is recommended to think about between-study variability in terms of the percent of variation that is due to heterogeneity among effect sizes rather than sampling error (i. e., $I^{2}$ ). Values equal to $25 \%, 50 \%$, and $75 \%$ are thought to reflect small, moderate, and large degrees of heterogeneity respectively (Higgins \& Thompson, 2002) and can be written 
as

$$
I^{2}=\frac{\tau^{2}}{\tau^{2}+v}
$$

With this information, the non-centrality parameter $\lambda$ can be calculated (see Eq. 7) and the cumulative distribution function of the test statistic can be obtained (Jackson \& Turner, 2017). Specifically, the cumulative distribution function of $\theta$ is given by

$$
P(T \leq t)=\Gamma_{1}\left(\frac{d f}{2}, \frac{d f\left(1-I^{2}\right)}{2}\right) \Phi\left((t-\lambda) \sqrt{1-I^{2}}\right)+2 d f \int_{\sqrt{\left(1-I^{2}\right)}}^{\infty} x \Phi\left(t x-\lambda \sqrt{1-I^{2}}\right) \chi_{d f}^{2}\left(d f x^{2}\right) d x
$$

where $I^{2}=\tau^{2} /\left(v+\tau^{2}\right), \lambda=E S / \sqrt{v / k}$ is the noncentrality parameter, and $\chi_{d f}^{2}(x)$ is the probability density function of the chi distribution with degrees of freedom, $d f=k-1$ (Jackson \& Turner, 2017). ${ }^{1}$ Since the probability of accepting the null hypothesis is given by $P\left(T \leq c_{\alpha}\right)$, power is given by subtracting this value from 1 , which is simply written as

$$
\text { power }=1-P\left(T \leq c_{\alpha}\right)
$$

For a two-tailed test, the logic is similar such that we evaluate the area under the curve that is either greater or less than $\left|c_{\alpha / 2}\right|$ written as

$$
\text { power }=1-P\left(T \leq c_{\alpha / 2}\right)-P\left(T \leq-c_{\alpha / 2}\right)
$$

\section{Power for test of homogeneity}

Meta-analysis software commonly reports a measure of homogeneity among the individual study effect sizes. The homogeneity statistic, Cochrane's $Q$, evaluates whether the amount of variation in individual effect sizes is greater than expected from just sampling error alone for fixedeffects models (Cochran, 1954). For random-effects models, $Q$ evaluates whether between-study variability $\tau^{2}$ is greater than 0 . As a result, statistical power can be computed for these tests given expectations about the amount of variation within (fixed-effects) and between (randomeffects) a group of studies.

\section{Fixed-effects model}

Computing power for a test of homogeneity requires an expectation of how much the individual effect sizes differ from the overall effect size (Pigott, 2012). This expectation can be instantiated as an alternative distribution, which can be compared to the null distribution to calculate the statistical power of the test. This alternative distribution is given by a non-centrality parameter, $\lambda$, written as

$$
\lambda=\sum_{i=1}^{k} w_{i}\left(\theta_{i}-\theta\right)^{2}
$$

where $\theta$ is the overall effect size, $\theta_{i}$ are the study-specific effects sizes, and $w$ is common inverse variance weight, $w=1 / v$. In this context, $\lambda$ depends on the difference between the study-specific effect sizes and the overall effect size (i. e., $\theta_{i}-\theta$ ). Since this is unknown prior to data collection, values for this difference must be provided. Pigott (2012) shows this can be done by positing the average difference between the study-specific effect sizes and the overall effect size in terms of standard deviation units (i. e., $\left.\sqrt{v_{\bullet}}\right)$. In this case, $\lambda$ can be rewritten replacing $\theta_{i}-\theta$ with the expected standard deviation, $s d$, which ultimately becomes just the square of the standard deviation shown here

$$
\begin{aligned}
\lambda & =\sum_{i=1}^{k} w\left(s d \sqrt{v_{\bullet}}\right)^{2} \\
& =k w v_{\bullet}(s d)^{2} \\
& =\frac{k v \bullet(s d)^{2}}{v} \\
& =\frac{k}{v} \times \frac{v}{k}(s d)^{2} \\
& =s d^{2}
\end{aligned}
$$

The test of homogeneity is based on $Q$, which follows a chi-square distribution and evaluates the amount of variation across study-specific effect sizes (Hedges \& Pigott, 2004). Here, the null hypothesis is that all effect sizes estimate a common effect $\left(H_{0}: \theta_{1}=\ldots=\theta_{k}=\theta\right)$. An alternative is that the study-specific effect sizes differ by some value of $s d$, which is reflected in the computation of $\lambda$ (see Eq. 15). Therefore, the statistical power for this test is the area under the alternative distribution with mean $\lambda$ that that exceeds $c_{\alpha}$ from a non-central chi-square distribution with a non-centrality parameter $\lambda$ and $k-1$ degrees of freedom written as

$$
\text { power }=1-F\left(c_{\alpha} \mid k-1 ; \lambda\right)
$$

\footnotetext{
${ }^{1}$ The lower incomplete gamma function is defined as $\Gamma_{1}(a, x)=\frac{1}{\Gamma(a)} \int_{0}^{x} t^{a-1} e^{-t} d t$.
} 


\section{Random-effects model}

For random-effects models, the test of homogeneity evaluates whether there is significant between-study variability among the study-specific effect sizes (i. e., $\tau^{2} \neq 0$ ). Given that $\tau^{2}$ and $I^{2}$ are related, Eq. 10 can be rewritten as $\tau^{2}=I^{2} v / 1-I^{2}$ to derive values of $\tau^{2}$ from different values of $I^{2}$ based on the expected degree of heterogeneity among effect sizes. Like fixed-effects models, the $Q$ statistic has a chi-square distribution with $k-1$ degrees of freedom; however, when the null hypothesis is false, the $Q$ distribution is a weighted combination of chi-square distributions, which must be approximated. Hedges and Pigott (2004) derived one way of approximating this distribution. First, the mean of the $Q$ distribution is given by

$$
\mu_{Q}=c \tau^{2}+(k-1)
$$

where $c$ is derived from the fixed-effects weights given by

$$
c=\sum_{i=1}^{k} w_{i}-\frac{\sum_{i=1}^{k} w_{i}^{2}}{\sum_{i=1}^{k} w_{i}}
$$

The variance of the $Q$ distribution is given by

$$
\sigma_{Q}^{2}=2 d f+4\left(\sum w_{i}-\frac{\sum w_{i}^{2}}{\sum w_{i}}\right) \tau^{2}+2\left(\sum w_{i}^{2}-2 \frac{\sum w_{i}^{3}}{\sum w_{i}}+\frac{\left(\sum w_{i}^{2}\right)^{2}}{\left(\sum w_{i}\right)^{2}}\right) \tau^{2}
$$

To compute the correct approximation with a central chi-square distribution with non-integer degrees of freedom, we must also compute $r$ and $s$ with

$$
\begin{gathered}
r=\sigma_{Q}^{2} / \mu_{Q} \\
s=2\left(\mu_{Q}\right)^{2} / \sigma_{Q}^{2}
\end{gathered}
$$

For this statistical test, the null hypothesis is that there is zero between-study variability (i. e., $H_{0}: \tau^{2}=0$ ). The alternative $\left(H_{1}: \tau^{2}>0\right)$ is derived based on the expected degree of heterogeneity (i. e., $I^{2}$ ). Therein, statistical power is the area under the alternative distribution that exceeds the critical value when $\tau^{2}=0$, given by

$$
\text { power }=F\left(c_{\alpha} / r \mid s ; 0\right)
$$

where $F(x)$ is the cumulative distribution function of the central chi-square distribution with $s$ degrees of freedom, and $c_{\alpha}$ is the $100(1-\alpha)$ percentile point of the chi-square distribution with $(k-1)$ degrees of freedom (Pigott, 2020).

\section{Power for moderator analysis}

Heterogeneity in meta-analysis is inevitable because studies are methodologically diverse. A recent study estimated that across 200 meta-analyses, including 12,065 effect sizes, the mean $I^{2}$ value was $74 \%$, which reflects a high degree of heterogeneity (Stanley, Carter, \& Doucouliagos, 2018). To investigate heterogeneity, moderator analyses are conducted to understand variation in the summary effect size. Moderator variables are defined for different groups of studies (e.g., studies that used different types of tasks). When the primary goal is moderator analysis, power anal- ysis is esepcially relevant to study feasibility because moderator analyses require more studies than simply estimating a summary effect size.

For moderator analysis, the goal is to compare the effect sizes for between different types of studies $p$ (e.g., children studies vs. adult studies; Hedges \& Pigott, 2004; Pigott, 2012). In this case, the number of studies, $k$, can be divided into different groups of studies: $k=m_{i}+\ldots+m_{p}$, where $i=1, \ldots, p$. Here, $m_{i}$ represents the number of studies that contribute to each level of a moderator variable. For power analysis, the individual values for $m_{i}$ are unknown; therefore, it is commonly assumed that an equal number of studies contribute to each group (e.g., the number of studies are divided evenly among levels of the moderator variable). In meta-analysis, moderator analysis is, in essence, analogous to a one-way analysis of variance, where the null hypothesis is that the overall effect size is equal across all groups $\left(\theta_{1}=\theta_{2}, \ldots,=\theta_{p}\right)$, whereas the alternative is that at least one group differs (Pigott, 2012). Power can be computed for a between-groups omnibus test of homogeneity to detect this alternative hypothesis. This requires posited values for the number of groups defined by the moderator effect sizes for each group, within-study sample size, total number of studies, and degree of between-study variability.

To compute power, the first step is to specify the number of groups, $p$, defined by a moderator variable. Metaanalyses commonly evaluate moderator variables related to age group (e.g., children, adolescents, and adults). Since moderator analyses evaluate between-study differences in effect sizes, $m_{i}$ designates the number of studies in the $i$ th group so that $k=m_{1}+m_{2}+\ldots+m_{p}$. Next, all group effect 
sizes $\theta_{i}$ must be specified for $i$ th group where $i=1, \ldots, p$, and the overall effect size $(\theta)$ should be calculated. For example, if is was expected that effect sizes were different for children, adolescents, and adults $\left(\theta_{\text {children }}=0.2\right.$, $\left.\theta_{\text {adolescents }}=0.4, \theta_{\text {adults }}=0.8\right)$, the overall effect size $\theta$ would be equal to $(0.2+0.4+0.8) / 3=0.47$ across these three groups. We must also assume that that each group is represented equally across studies (Pigott, 2012). For example, for a meta-analysis of 30 studies, each group would be divided evenly among the total number of studies, $k$, where $m_{\text {children }}=m_{\text {adolescent }}=m_{\text {adult }}=10$.

The next step is to compute the inverse-variance weights for each group separately computed with

$$
w_{i}=\sum_{j=1}^{m_{i}} w_{i j}
$$

where $i=1, \ldots, p$, and $j=1, \ldots, m_{i}$, and $w_{i j}=1 / v_{i j}$. With these values, an expected alternative (i. e., at least one group effect size is different) can be derived by calculating the non-centrality parameter $\lambda$ given by

$$
\lambda=\sum_{i=1}^{p} w_{i}\left(\theta_{i}-\theta\right)^{2}
$$

where $p$ is the number of groups, $w_{i}$ are the summed weights, $\theta_{i}$ are the expected group effect sizes, and $\theta$ is the overall effect size. Subsequently, the power for this test is written as

$$
\text { power }=F\left(c_{\alpha} \mid p-1 ; \lambda\right)
$$

where $F(x)$ is the cumulative distribution of the noncentral chi-square with $p-1$ degrees of freedom and noncentrality parameter $\lambda$. Statistical power for this test reflects the area under the curve that exceeds the critical value $c_{\alpha}$.

\section{Random-effects model}

The same sequence of steps applies to random-effects models with one exception. The between-study variability, $\tau^{2}$ must be incorporated into the variance estimate. The conventional method for this is to represent the variance as the sum of the variance, $v_{i j}^{2}$, and between-study variability, $\tau^{2}$ (Pigott, 2012). Here, the inverse variance weights can be written as

$$
w_{i}=\sum_{j=1}^{m_{i}} \frac{1}{\left(v_{i j}^{2}+\tau^{2}\right)}
$$

Subsequently, the non-centrality parameter and power can be computed in the same way as the fixed-effects method (see Eq. 24 \& 25).

\section{Subgroup analysis}

In addition to evaluating group differences between studies, group differences within studies are commonly evaluated with subgroup analysis. For instance, meta-analysts may be interested in how the summary effect differs among different subgroups of study samples, like men and women. To evaluate power for subgroup analysis, the basic logic is similar to that of power for moderator analysis (Pigott, 2020). The main difference is that the number of groups, $p$, now reflects the number of subgroups within a study. As a result, the common variance $v$ is now calculated for both subgroups instead of the entire study sample. For parsimony, we make the simplifying assumption that the subgroups are represented equally in each study $\left(N=40 ; n_{\text {men }}=20, n_{\text {women }}=20\right)$. Additionally, since the subgroup differences are within-study comparisons, the number of studies $k$ is not divided among groups as in the moderator analysis; therefore, $k=m_{1}=m_{2}=\ldots=m_{p}$. Aside from these specifications, power analysis for subgroups can be calculated using the same sequence as the moderator analysis (see Eq. 23-26).

\section{Determining parameter values for power analysis}

As outlined above, calculating statistical power is a complex procedure that requires postulations about expected values such as the effect size, study size, number of studies, and degree of statistical heterogeneity. Importantly, these values have a large impact on statistical power and should not be arbitrarily selected. Instead, these expected values should be informed decisions based on experience and previous literature. Furthermore, expected values are reasonable guesses which are not always correct; it is thus important to be transparent and open about the process of arriving at these expected values. In what follows, I provide guidance on making these informed decisions.

\section{Effect Size Magnitude}

The expected effect size has a significant impact on statistical power such that large effect sizes require fewer studies to obtain the same power as small effect sizes. Instead of selecting arbitraily, the expected effect size should be informed by likelihood constraints, previous literature, and experience. For example, although Cohen's $d$ ranges from 0 to $\infty$, the average effect size in psychology is 0.4 with $87 \%$ of all effects being less than 0.8 (Cumming, 2014). Therefore, in most psychology applications, the suggested benchmarks of small (Cohen's $d=0.2$ ), moderate (Cohen's $d=0.5$ ), and large (Cohen's $d=0.8$ ) are good starting points. However, the expected effect size should be directly informed by previous literature when possible.

Unlike primary research, previous literature is a pre- 
requisite to meta-analysis; therefore, access to previous estimates of a particular effect from individual studies are always available. For example, while planning a metaanalysis, a brief examination of the literature can provide some estimates of a particular effect. Note that studies will not always report effect size information, but they can be calculated manually (see Eq. 1, 3, 5). If numerous studies report a Cohen's $d$ between 0.2 and $0.6,0.5$ would be a reasonable guess for the expected effect size of a metaanalysis. In contrast, in some areas of psychology, like psychopharmacology, effect sizes can be considerably larger (Cohen's $d=5$ ). It is also important to consider that the published literature tends to overestimate the true magnitude of effects due to publication bias, or the tendency for articles to be published based on statistical significance (Gelman \& Carlin, 2014). In sum, researchers evaluating the feasibility of a prospective meta-analysis can make informed judgments based on previous estimates and general knowledge of the published literature.

\section{Study Size}

Power analysis also requires expected values of the "typical" sample size of a study to be included in the metaanalysis, which varies considerably across different research areas (e.g., Hedges \& Pigott, 2001; Jackson \& Turner, 2017). For this expected value, area-specific contextual knowledge can provide reasonable guesses about the typical study size. For example, for research questions that can be addressed with standard questionnaires in large online studies, study sizes may be considerably large (e.g., $N$ = 500); however, research questions related to those with neurodevelopmental disorders are likely much smaller (e.g., $N=40$ ). Furthermore, there can be considerable variability in study sizes among studies in a specific area of research. For example, studies of neurodevelopmental disorders are generally small (e.g., $N=40$ ), but studies evaluating specific subgroups of the population (e.g., those with a co-morbid condition) or using a specific methodology (e.g., eye-tracking) can be even smaller (e.g., $N=20$ ). Therefore, the expected study size should be highly specific to the meta-analytic research question.

\section{Number of Studies}

How many studies are needed to conduct a meta-analysis? The answer lies at the core of power analysis: to determine if a given research question has enough published studies to warrant conducting a meta-analysis. As shown in the previous section of the paper, the number of studies required for adequate meta-analytic power depends on the expected effect size, study size, Type 1 error probability, test directionality (1-tailed vs. 2-tailed), type of statistical test (e.g., summary effect size, moderator analysis), and model assumptions (fixed- vs. random-effects model). For instance, under a fixed-effects model, which assumes that variability in effect sizes is purely due to sampling error, the number of studies needed to conduct a metaanalysis that has more power than an individual study is two (Valentine et al., 2009). However, in psychology, the fixed-effects assumption is rarely justified and difficult to defend; therefore the random-effects model, which assumes that effect size variability is due to sampling variability and between-study differences, is widely used. Under the random-effects model, Jackson and Turner (2017) proposed a general rule of thumb that at least five studies are required for a meta-analysis that is more informative than the largest individual study of that meta-analysis.

Fortunately, before researchers conduct a metaanalysis, there is generally some notion regarding the size of the published literature. For instance, published narrative or systematic reviews of the literature make estimating the total number of published studies trivial. However, for newer areas of research, where fewer studies have been published, it can be difficult to anticipate the total number of published studies on a given research question. If a previous systematic review of the literature included 40 empirical studies, it is reasonable to assume that at least 40 studies will be included in a meta-analysis. However, in a new area of research where the meta-analyst is only aware of 5 empirical studies, and no previous reviews have been conducted, it may be reasonable to assume that 5-10 studies will be included in the meta-analysis. For randomized controlled trials, which are commonly registered publicly (e.g., clinicaltrials.gov), researchers can proactively search these databases to get an idea of how many studies would be included in a meta-analysis. Likewise, a preliminary search of journal databases can provide an approximation for the number of total studies. In sum, determining the size of a literature pertinent to a specific metaanalytic research question may require extensive knowledge of the field or a brief search of the literature to arrive at a reasonable guess for the anticipated number of studies.

\section{Statistical Heterogenity}

In addition to these expected quantities, statistical power is greatly impacted by between-study variation in effect sizes (i. e., $\tau^{2}$ ). For example, more data are required for metaanalyses with highly variable effect sizes to achieve the same statistical power as those with highly homogeneous effect sizes. Theoretically, between-study variation can be incorporated to estimate statistical power, but values of $\tau^{2}$ are difficult to interpret and anticipate. As a result, estimates of $\tau^{2}$ can be estimated from another index of heterogeneity (i. e., $I^{2}$; Higgins \& Thompson, 2002). The $I^{2}$ 
statistic is a simple and intuitive index of statistical heterogeneity that quantifies the percentage of variation across studies that is not due to sampling variability (see Eq. 10).

For fixed-effects models, all variation in effect sizes is attributed to sampling error and thus $I^{2}=0$. However, this assumption is only justified in very specific situations. In the wake of the replication crisis, there has been increased emphasis on conducting direct replications. Replication studies attempt to replicate the methodology and results of a previous study in a new sample. Here, it may be reasonable to assume that all studies were measuring a single common effect size, and calculate statistical power under a fixed-effects model (i. e., assuming that $I^{2}=0$ ). Another example may be the meta-analysis of multiple randomized controlled trials of the same treatment. It is common for there to be multiple studies evaluating the efficacy of a specific treatment for a specific medical condition; in these situations, a fixed-effects model may also be justified given the homogeneity in methodology and implementation.

The vast majority of meta-analyses are conducted under a random-effects model, which assumes that some percentage of between-study variation is not due to sampling error (i. e., $I^{2}>0$ ). Anticipating values of $I^{2}$ can be difficult, but standard conventions of $I^{2}$ values equal to $25 \%$, $50 \%$, and $75 \%$ reflect small, moderate, and large degrees of heterogeneity have been established (Higgins \& Thompson, 2002). In addition, recent evidence across 200 metaanalyses in psychology, including 12,065 effect sizes, show that the average $I^{2}$ value was $74 \%$ (Stanley et al., 2018), indicating large heterogeneity among effect sizes was common. Thus, unless researchers have a strong prior belief that heterogeneity will be small, a conservative approach would be to expect a moderate to high degree of heterogeneity as reflected in $I^{2}$ values of $50 \%$ or $75 \%$. In sum, all of aforementioned parameter values should be informed by previous literature when possible, but there are some general rules of thumb when informed decisions cannot be made.

\section{Tutorial: Using metapower to calculate statistical power for meta-analysis}

metapower is a freely available open source $\mathrm{R}$ package. All power analysis calculations were derived from the most recent theoretical statistics and methodology. This includes power analysis for summary effect sizes (Hedges \& Pigott, 2001; Jackson \& Turner, 2017), test of homogeneity (Pigott, 2012), moderator analysis (Hedges \& Pigott, 2004), and subgroup analysis (Pigott, 2020). All source code is publicly available on github (github.com/jasonwgriffin/metapower). metapower can be downloaded in R (cran.r-project.org) or Rstudio (rstudio.com). Users must have R version 3.6.0 or later. Because metapower is hosted on the Comprehensive R Archive Network (CRAN.R-project.org/package=metapower), it can be downloaded and attached directly in Rstudio using

install.packages ("metapower")

library (metapower)

\section{Example Research Question}

Researchers are often interested in using meta-analysis to quantify the group difference in some outcome (e.g., working memory, face recognition, processing speed) between two independent groups (e.g., clinical population vs. typically developing, men vs. women). Since this type of research question reflects the mean difference between two independent groups on some outcome measure, it would be most appropriate to compute power based on Cohen's $d$. For this example, we will assume a previous review of relevant literature included 20 studies, that had study sizes around 40, and routinely reported effect sizes around 0.4. In addition, the outcome was measured across a wide variety of tasks; therefore, there is likely to be considerable statistical heterogeneity across studies (e.g., $I^{2}=75 \%$ ). With this contextual knowledge, we can compute statistical power given these expected values.

\section{Summary effect size}

To calculate statistical power for detecting a mean difference between between the two groups, use the mpower ( ) function with the aforementioned expected values while specifying the effect size type (es_type $=d$ ) as shown below

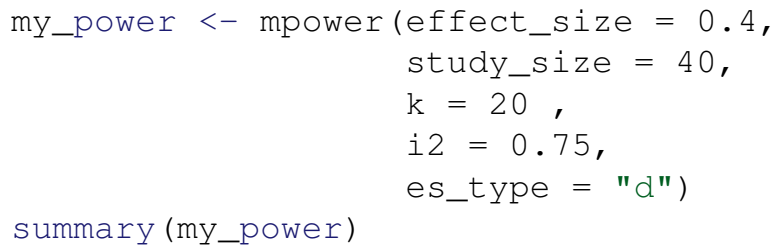

The first section of the output shows information about the user-specified input parameters. Note that for Cohen's $d$ the study size reflects the entire sample, which are di- 
Figure 1 - Power curves generated from plot_power ( ) for estimating a summary effect size. Power curves reflect statistical power as a function of the number of studies, $k$. By default, power curves are shown for the user-specified effect size under a fixed- and random-effects model. The range of values for the number of studies axis is 5 times that specified by the user. The point along the power curve reflects the current power estimate given the user-specified input parameters. The dashed horizontal line reflects $80 \%$ power.

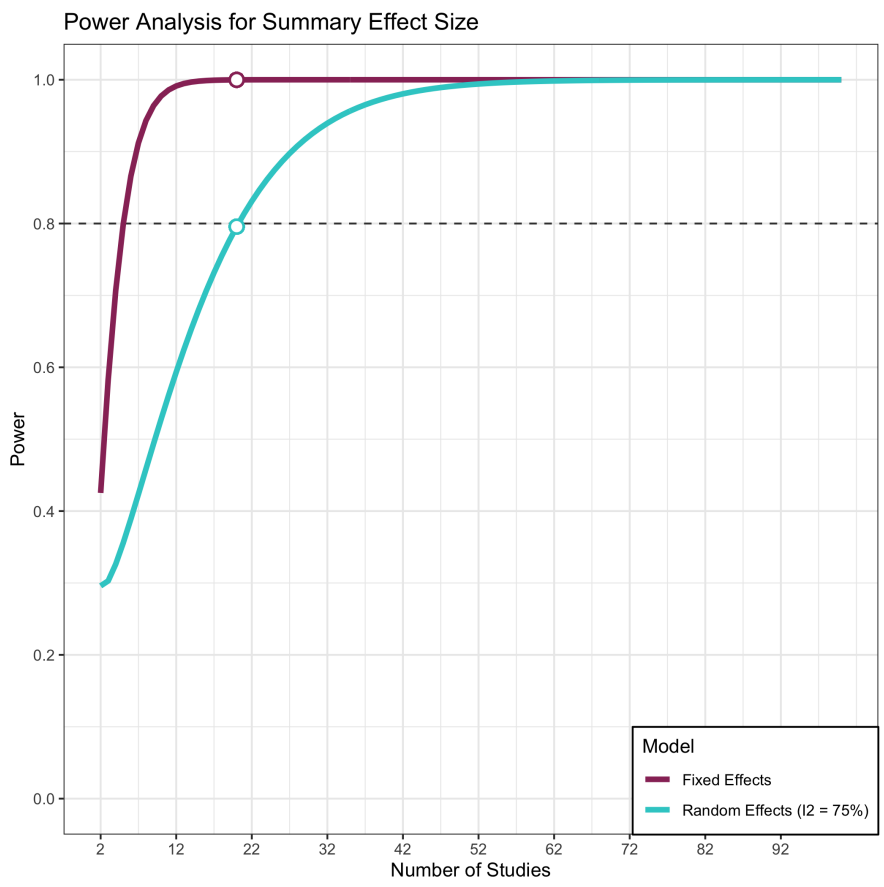

can access the web-based application to set parameters according to their research question and calculate statistical power for each of the statistical tests described here as well as visualize the respective power curves (see Figure 5).

\section{Discussion}

Power analysis plays an important role in determining the feasibility of prospective meta-analyses. Despite this, power analyses are rarely conducted in published metaanalyses. Two major barriers to the routine inclusion of power analyses in meta-analysis include: the complexity of calculating statistical power and the absence of available software for carrying out such power calculations. As demonstrated, power calculations are conditional on a number of different values, which include the total number of studies, study sizes, effect size magnitude, effect size metric, model assumptions, test directionality, Type 1 error probability, and degree of statistical heterogeneity. These values must be used in a complex series of formulas and equations for power to be calculated. metapower provides a tool for researchers to instantiate this complexity in just a single line of code.
In this tutorial, I have shown how metapower can be used to appropriately plan a meta-analysis by conducting an apriori power analysis without necessitating a deep knowledge of statistics or programming. Furthermore, metapower offers an effective and efficient solution to address the common question, "Is the literature large enough to warrant the time and effort it takes to conduct a metaanalysis?" It accomplishes this in a way that is computationally simple, easy-to-use, and entirely reproducible. metapower can also be integrated into a single workflow with other R packages. For example, numerous packages exist for the major steps of any meta-analysis, which include searching the literature, screening articles, and analyzing the data. metapower is another tool used to determine when a meta-analysis is warranted. As an added bonus, users unfamiliar with $\mathrm{R}$ can access a fully functional, web-based shiny application to implement the core functions of metapower using a graphical user interface. This shiny application requires no programming experience and can be opened in any web browser. 
Figure 2 - Power curves generated from plot_homogen_power ( ) . Power curves reflect statistical power as a function of the number of studies, $k$. The top panel displays power curves under a fixed-effects model. Power curves are shown for various levels of variation among effect sizes (i. e., SD among study-specific effect sizes). The bottom panel reflects the power curve under a random-effects model with the user-specified heterogeneity. The points along the power curve reflects the current power estimate given the user-specified input parameters The dashed horizontal line reflects $80 \%$ power.
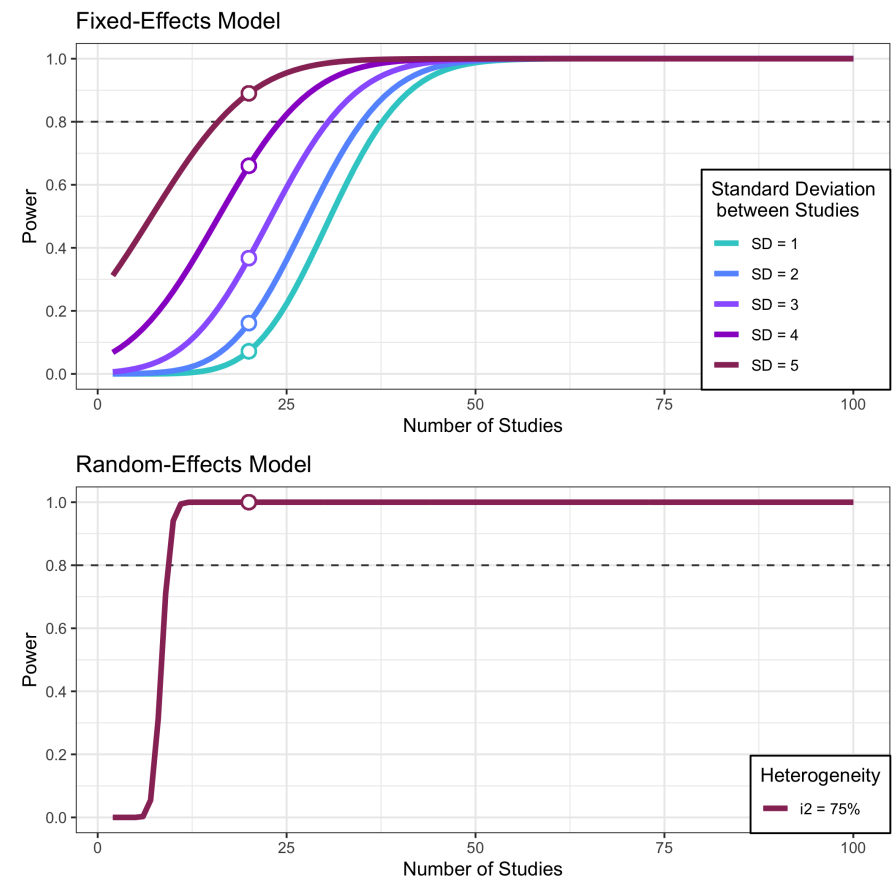

\section{Limitations}

Statistical power fundamentally depends on expecting the actual presence of an effect. That is, primary studies are conducted with the expectation of finding a significant difference between two groups or a significant association between two variables. However, in meta-analysis, we may actually expect that a summary effect size will not be statistically different than zero. In this way, meta-analyses sometimes demonstrate that an effect size is not distinguishable from zero despite individual studies suggesting otherwise. Currently, metapower is not capable of computing statistical power for tests of equivalence, which are are used to statistically reject the presence of an effect (Goertzen \& Cribbie, 2010; Lakens, 2017; Lakens, Scheel, \& Isager, 2018). Given that equivalence tests are simply a reformulation of the standard null-hypothesis significance testing framework, it is possible to calculate statistical power for these types of statistical tests for primary research (e.g., Shieh, 2016; Lakens, 2017). In theory, these power calculations could be adapted for meta-analysis in the future.
Similarly, statistical power is inextricably associated with specific statistical tests that are focused on rejecting the null hypothesis (e.g., $H_{0}=0$ ). However, researchers may not be interested in simply showing that an effect size is different than zero. For instance, it can be valuable to power a study capable of estimating an effect size within a specific margin of error. One reason for this would be that a clinically significant effect in some context would only be one that was at or above a certain value. In this case, it would be useful to power a meta-analysis for precision - as opposed to statistical power - in order to estimate a confidence interval that was above a certain value. Given that these methods are being developed in primary research (see Goulet-Pelletier \& Cousineau, 2018), they may be adapted to meta-analysis in the future.

As it relates to moderator and subgroup analyses, metapower assumes that categorical groups (e.g., moderator or subgroup analyses) are equal in number of studies and sample size. For situations where there are unequal group sizes, the power estimates provided by metapower will overestimate statistical power. However, given that 
Figure 3 - Power curves generated from plot_mod_power ( ). Power curves reflect statistical power as a function of the number of studies, $k$. Power curves reflecting the power to detect between-study differences (categorical moderators) among studies are presented under a fixed- and random-effects model. The points along the power curve reflects the current power estimate given the user-specified input parameters The dashed horizontal line reflects $80 \%$ power.

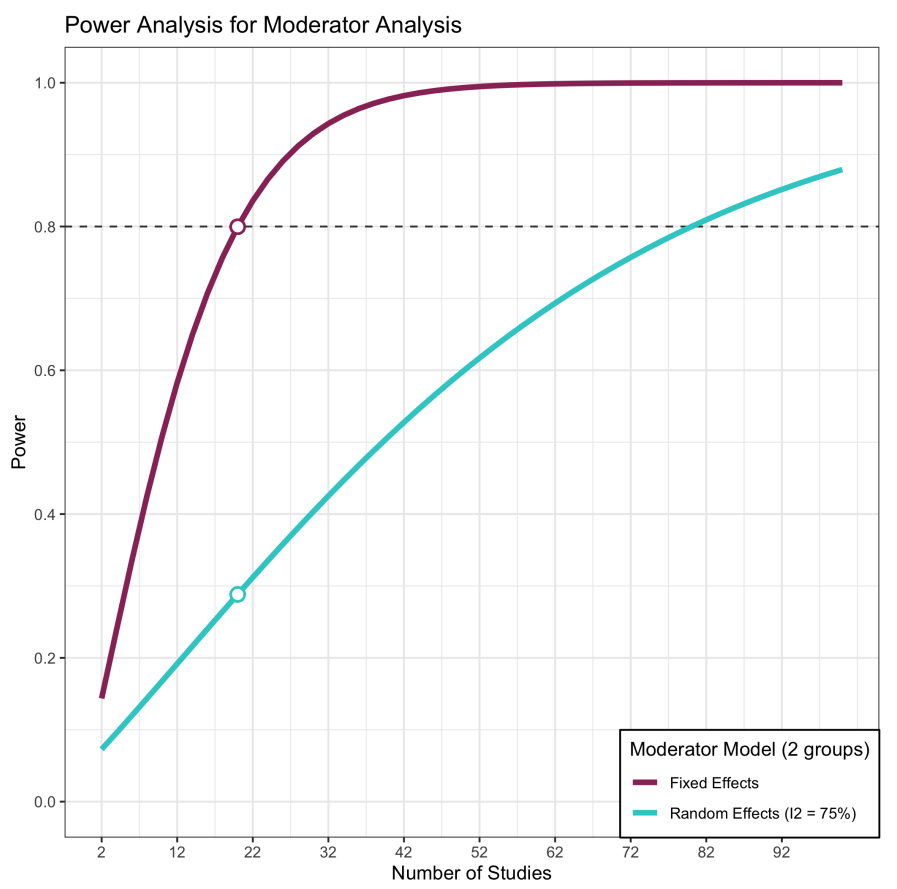

power analyses are conducted apriori, it is untenable to make guesses about how many studies will comprise a particular category. In addition, metapower is limited in that all calculations assume that each study contributes a single effect size to the meta-analysis. For meta-analyses that incorporate hierarchical structure (i. e., multiple effect sizes from a study), power cannot currently be calculated for these more complex data structures.

\section{Future Development}

metapower currently accommodates the three most commonly used effect sizes in meta-analysis (i. e., Cohen's $d$, correlation, and odds ratio); however, future versions of metapower plan to incorporate additional effect size metrics to handle these research designs (see Goulet-Pelletier \& Cousineau, 2018). For example, metapower currently handles Cohen's $d$ for between-subject designs, but future developments can include calculations for Cohen's $d$ in one-sample or correlated measurements (e.g., Lakens, 2013). Additionally, multilevel modeling (e.g., two- and three-level models) is becoming an increasingly popular method for meta-analysis since it can include multiple effect sizes from source studies while modeling the statisti- cal dependence among observations (Assink \& Wibbelink, 2016). These models achieve more statistical power than traditional methods, but there is no agreed upon method for calculating statistical power for these more complex models. Future versions of metapower will incorporate these more advanced power caluclations as the methods become available and validated.

\section{Conclusion}

The influence of meta-analyses in the scientific community is ubiquitous. Given their impact, high-quality metaanalyses should be conducted when there are a sufficient number of published studies given a particular research question. To do this objectively, power analysis specific to the research question (summary effect size, moderator analysis, subgroup analysis) should be conducted during the planning phase of a prospective meta-analysis. To my knowledge, metapower is the first freely available and easy-to-use software package that allows researchers to do this. 
Figure 4 ॥ Power curves generated from plot_subgroup_power ( ) . Power curves reflect statistical power as a function of the number of studies, $k$. Power curves reflecting the power to detect within-study differences (subgroups) among studies are presented under a fixed- and random-effects model. The points along the power curve reflects the current power estimate given the user-specified input parameters The dashed horizontal line reflects $80 \%$ power.

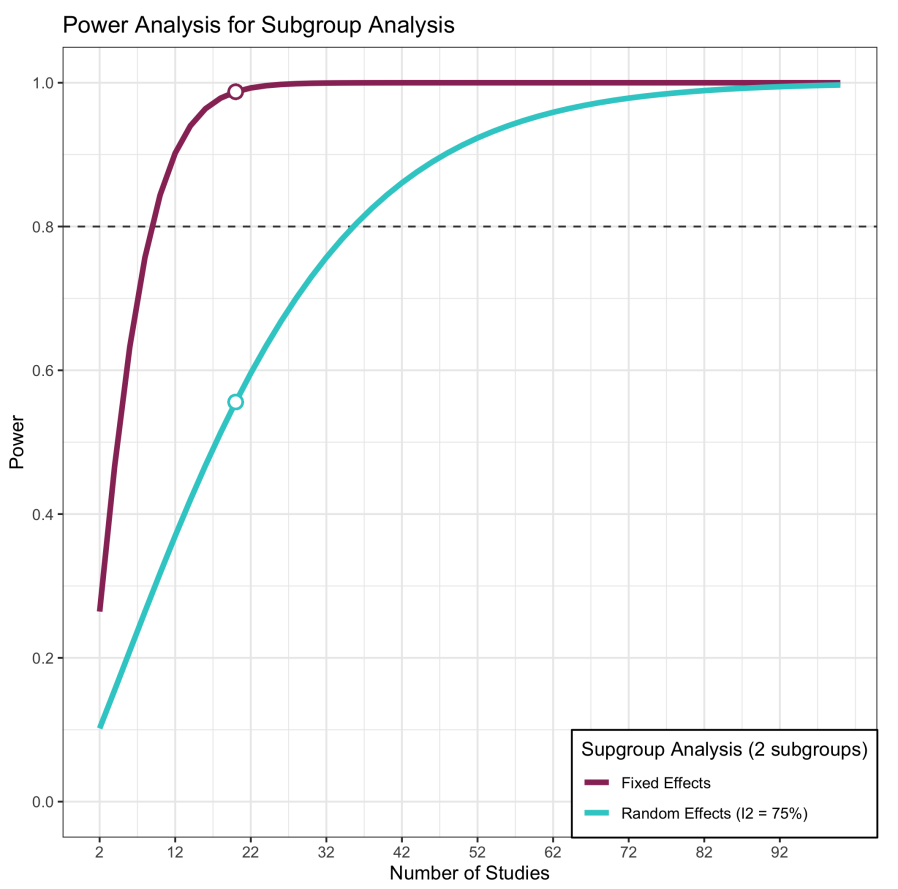

\section{Authors' note}

I would like to thank Flora Oswald and Courtney R. Gerver for their feedback on the manuscript.

\section{References}

Assink, M., \& Wibbelink, C. J. M. (2016). Fitting three-level meta-analytic models in r: A step-by-step tutorial. The Quantitative Methods for Psychology, 12(3), 154-174. doi:10.20982/tqmp.12.3.p154

Borah, R., Brown, A. W., Capers, P. L., \& Kaiser, K. A. (2017). Analysis of the time and workers needed to conduct systematic reviews of medical interventions using data from the prospero registry. BMJ Open, 7(2), e012545. doi:10.1136/bmjopen-2016-012545

Cafri, G., Kromrey, J. D., \& Brannick, M. T. (2009). A sas macro for statistical power calculations in metaanalysis. Behavior Research Methods, 41(1), 35-46. doi:10.3758/brm.41.1.35

Cochran, W. G. (1954). The combination of estimates from different experiments. Biometrics, 10(1), 101-129. Retrieved from http://www.jstor.org/stable/3001666
Cumming, G. (2014). The new statistics: Why and how. Psychological Science, 25(1), 7-29. doi:10 . 1177 / 0956797613504966

Davey, J., Turner, R. M., Clarke, M. J., \& Higgins, J. P. (2011). Characteristics of meta-analyses and their component studies in the cochrane database of systematic reviews: A cross-sectional, descriptive analysis. $B M C$ Medical Research Methodology, 11(1), 160-170. doi:10. 1186/1471-2288-11-160

Faul, F., Erdfelder, E., Lang, A.-G., \& Buchner, A. (2007). $\mathrm{G}^{*}$ power 3: A flexible statistical power analysis program for the social, behavioral, and biomedical sciences. Behavior Research Methods, 39(2), 175-191. doi:10.3758/bf03193146

Gelman, A., \& Carlin, J. (2014). Beyond power calculations. Perspectives on Psychological Science, 9(6), 641-651. doi:10.1177/1745691614551642

Goertzen, J. R., \& Cribbie, R. A. (2010). Detecting a lack of association: An equivalence testing approach. British Journal of Mathematical and Statistical Psychology, 63(3), 527-537. doi:10.1348/000711009x475853

Gopalakrishnan, S., \& Ganeshkumar, P. (2013). Systematic reviews and meta-analysis: Understanding the best 
Figure 5 - Shiny application interface for metapower. The top menu tabs correspond to the different types of power analysis, including summary effect size, test of homogeneity, moderator analysis, and subgroup analysis. The left panel controls the expected input parameters. The right panel displays the power curves under the Power Curve panel (default), but can also view the summary statistical power results using the Summary tab.

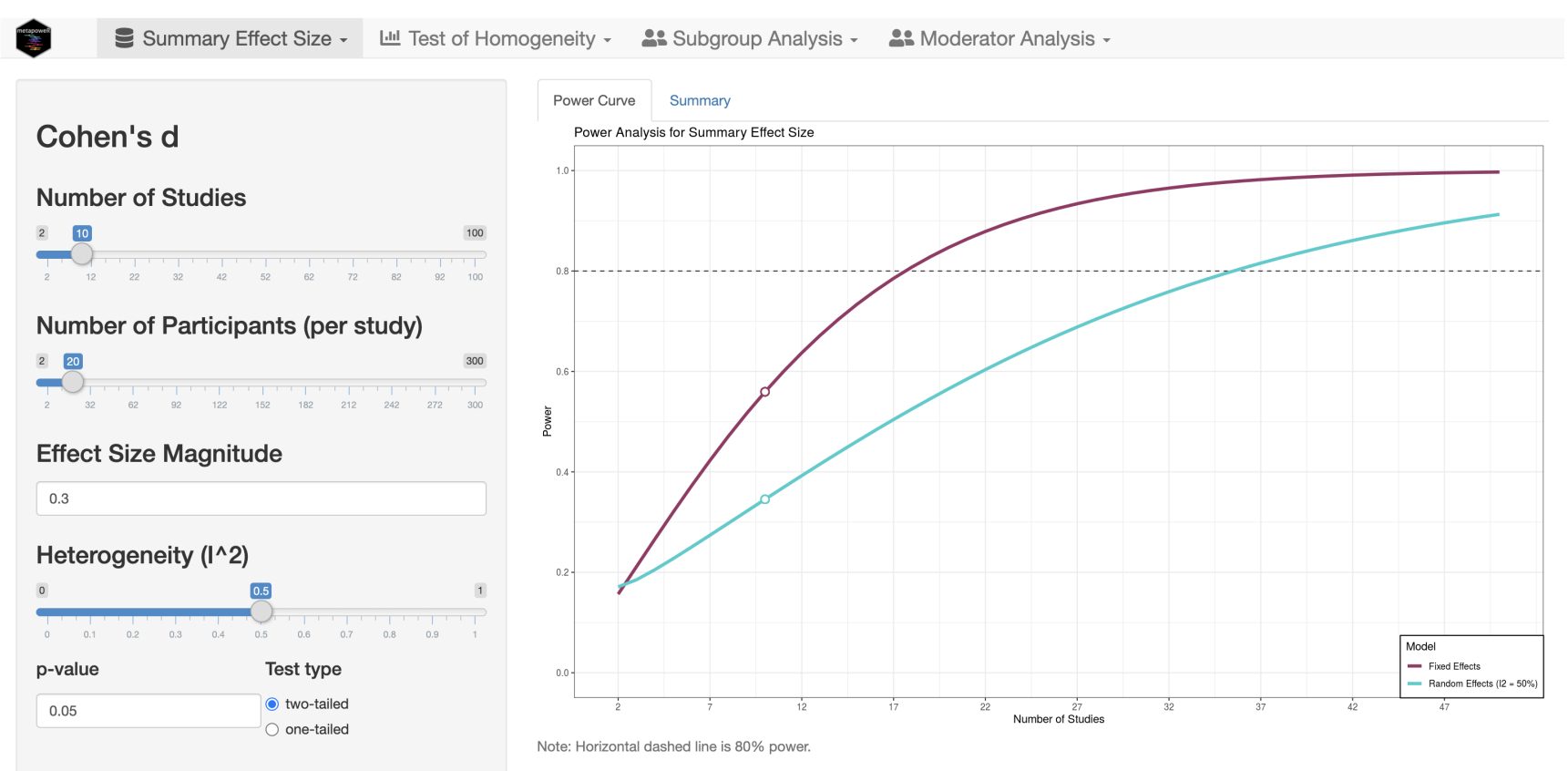

evidence in primary healthcare. Journal of Family Medicine and Primary Care, 2(1), 9-14. doi:10.4103/ 2249-4863.109934

Goulet-Pelletier, J.-C., \& Cousineau, D. (2018). A review of effect sizes and their confidence intervals, part I: The Cohen's d family. The Quantitative Methods for Psychology, 14(4), 242-265. doi:10.20982/tqmp.14.4.p242

Hedges, L. V., \& Pigott, T. D. (2001). The power of statistical tests in meta-analysis. Psychological Methods, 6(3), 203-217. doi:10.1037/1082-989x.6.3.203

Hedges, L. V., \& Pigott, T. D. (2004). The power of statistical tests for moderators in meta-analysis. Psychological Methods, 9(4), 426-445. doi:10.1037/1082-989x.9.4.426

Higgins, J. P. T., \& Thompson, S. G. (2002). Quantifying heterogeneity in a meta-analysis. Statistics in Medicine, 21(11), 1539-1558. doi:10.1002/sim.1186

Jackson, D., \& Turner, R. (2017). Power analysis for random-effects meta-analysis. Research Synthesis Methods, 8(3), 290-302. doi:10.1002/jrsm.1240

Lakens, D. (2013). Calculating and reporting effect sizes to facilitate cumulative science: A practical primer for ttests and anovas. Frontiers in Psychology, 4, 863-893. doi:10.3389/fpsyg.2013.00863
Lakens, D. (2017). Equivalence tests: A practical primer for t tests, correlations, and meta-analyses. Social Psychological and Personality Science, 8(4), 355-362. doi:10. 1177/1948550617697177

Lakens, D., Scheel, A. M., \& Isager, P. M. (2018). Equivalence testing for psychological research: A tutorial. Advances in Methods and Practices in Psychological Science, 1(2), 259-269. doi:10.1177/2515245918770963

National Institute of Health. (2018). Enhancing reproducibility through rigor and transparency. Retrieved from https://grants.nih.gov/policy/reproducibility/ index.htm

Pigott, T. D. (2012). Advances in meta-analysis. doi:10.1007/ 978-1-4614-2278-5

Pigott, T. D. (2020). Power of statistical tests for subgroup analysis in meta-analysis. In J. C. C. Ting, S. Ho, \& (. D.-G. Chen (Eds.), $N$ (pp. 347-368). Design: Springer International Publishing.

Pigott, T. D., \& Polanin, J. R. (2020). Methodological guidance paper: High-quality meta-analysis in a systematic review. Review of Educational Research, 90(1), 2446. doi:10.3102/0034654319877153 
Shieh, G. (2016). Exact power and sample size calculations for the two one-sided tests of equivalence. PLOS ONE, 11(9), e0162093. doi:10.1371/journal.pone.0162093

Stanley, T. D., Carter, E. C., \& Doucouliagos, H. (2018). What meta-analyses reveal about the replicability of psychological research. Psychological Bulletin, 144(12), 1325-1346. doi:10.1037/bul0000169

Thorlund, K., Imberger, G., Walsh, M., Chu, R., Gluud, C., Wetterslev, J., ... Thabane, L. (2011). The number of patients and events required to limit the risk of overestimation of intervention effects in meta-analysis? a simulation study. PLOS ONE, 6(10), e25491. doi:10. 1371/journal.pone.0025491

Valentine, J. C., Pigott, T. D., \& Rothstein, H. R. (2009). How many studies do you need? Journal of Educational and Behavioral Statistics, 35(2), 215-247. doi:10.3102/ 1076998609346961

\section{Citation}

Griffin, J. W. (2021). Calculating statistical power for meta-analysis using metapower. The Quantitative Methods for Psychology, 17(1), 24-39. doi:10.20982/tqmp.17.1.p024

Copyright (C) 2021, Griffin. This is an open-access article distributed under the terms of the Creative Commons Attribution License (CC BY). The use, distribution or reproduction in other forums is permitted, provided the original author(s) or licensor are credited and that the original publication in this journal is cited, in accordance with accepted academic practice. No use, distribution or reproduction is permitted which does not comply with these terms.

Received: 11/11/2020 Accepted: 16/02/2021 